\title{
Article
}

\section{The Prevalence of Limited Health Literacy and Its Associated Factors among Elderly Patients Attending an Urban Academic Primary Care Clinic in Malaysia}

\author{
Siti Nur Hidayah Abd-Rahim ${ }^{1}$ (D), Mohamed-Syarif Mohamed-Yassin 1,*(D), Suraya Abdul-Razak 1,2,3, \\ Mohamad Rodi Isa ${ }^{4}$ and Noorhida Baharudin ${ }^{1}$ (D)
}

1 Department of Primary Care Medicine, Faculty of Medicine, Universiti Teknologi MARA, Jalan Prima Selayang 7, Batu Caves 68100, Malaysia; adrenalina4c@yahoo.com (S.N.H.A.-R.); suraya617@uitm.edu.my (S.A.-R.); noorhida8229@uitm.edu.my (N.B.)

2 Institute of Pathology, Laboratory and Forensic Medicine (I-PPerForM), Universiti Teknologi MARA, Sungai Buloh 47000, Malaysia

3 Cardio Vascular and Lungs Research Institute (CaVaLRI), Pusat Perubatan UiTM, Kampus Sungai Buloh, Sungai Buloh 47000, Malaysia

4 Department of Public Health Medicine, Faculty of Medicine, Universiti Teknologi MARA, Jalan Hospital, Sungai Buloh 47000, Malaysia; rodi@uitm.edu.my

check for updates

Citation: Abd-Rahim, S.N.H.; Mohamed-Yassin, M.-S.;

Abdul-Razak, S.; Isa, M.R.; Baharudin, N. The Prevalence of Limited Health Literacy and Its Associated Factors among Elderly Patients Attending an Urban Academic Primary Care Clinic in Malaysia. Int. J. Environ. Res. Public Health 2021, 18, 9044. https:// doi.org/10.3390/ijerph18179044

Academic Editors: Kristine Sorensen, Duong Van Tuyen and Andrew Pleasant

Received: 30 July 2021

Accepted: 24 August 2021

Published: 27 August 2021

Publisher's Note: MDPI stays neutral with regard to jurisdictional claims in published maps and institutional affiliations.

Copyright: (c) 2021 by the authors. Licensee MDPI, Basel, Switzerland. This article is an open access article distributed under the terms and conditions of the Creative Commons Attribution (CC BY) license (https:// creativecommons.org/licenses/by/ $4.0 /)$.
* Correspondence: syarif8258@uitm.edu.my; Tel.: +60-36-126-4655

Abstract: Limited health literacy (HL) is linked to many negative health outcomes, including poor self-management of chronic diseases and medication adherence among patients. There are a lack of data regarding HL in the elderly population in Malaysia. This study aimed to determine the prevalence of limited HL levels and its associated factors among elderly patients in an urban academic primary care clinic in Selangor, Malaysia. A cross-sectional study was conducted among 413 elderly patients ( $\geq 60$ years old) who attended this academic primary care clinic between January 2020 and January 2021. Sociodemographic data, clinical characteristics, and health literacy scores were collected. Descriptive statistics (median with interquartile ranges (IQR), frequency, and percentages) and multiple logistic regression were utilized. The prevalence of limited HL in our population was $19.1 \%$ (95\% CI: $15.3,23)$. The middle-old (70-79 years) and very-old ( $\geq 80$ years) age groups were more likely to have limited HL (aOR 4.05; 95\% CI: 2.19, 7.52 and aOR 4.36; 95\% CI: 1.02, 18.63, respectively). Those with at least secondary school education (aOR 0.06; 95\% CI: 0.02, 0.24) and those who found medical information via the internet/television (aOR 0.21; 95\% CI: 0.05, 0.93) had lower odds of having limited HL. In conclusion, having limited HL levels was not common among elderly patients in this primary care clinic. Further studies involving rural and larger primary care clinics in Malaysia are required to support these findings.

Keywords: health literacy; elderly; older adult; aging; primary care; Malaysia

\section{Introduction}

Health literacy (HL) has been identified as a critical component to improve health and well-being while reducing health disparities [1]. The World Health Organization (WHO) defines HL as the "cognitive and social skills which determine the motivation and ability of individuals to gain access, understand and use information, which promotes and maintain good health" [1]. The purpose of HL is to help people make health-related decisions and take appropriate actions to manage their health $[1,2]$. The ability to find, understand, appraise, and apply information related to health is essential in order to prevent disease and promote good health $[1,2]$.

Limited HL is linked to increased healthcare problems, including medical and medication mistakes, poor adherence to prescribed medications, difficulty communicating 
with healthcare providers, and a lack of understanding of disease procedures and selfmanagement abilities in people with chronic diseases such as diabetes, hypertension, and heart disease [3-5]. Limited HL has also been found to weaken elderly people's autonomy and limit their independence $[4,6]$. Hence, empowering patients to make meaningful health decisions and participate in health-promoting and sustaining activities are critical for improving their health outcomes [7]. Better health outcomes and lower utilization of healthcare services are the outcomes of good HL levels in terms of cost efficiency, frequency, and length of hospitalization [8-10].

The elderly population makes up 30\% of the global population [11]. Most East Asian middle-income countries are aging at a rapid pace, faster than Europe and the United States did last century [12]. Over the last half-century, Malaysia's demographic age structure has changed significantly [13]. Malaysia follows the definition of the United Nations World Assembly on Ageing in Vienna in 1982 to define the elderly as those aged 60 and above [14]. Like the rest of the world, it is experiencing an increase in the number of elderly people $[13,15]$. This is attributable to a variety of causes, including lower fertility rates, lower infant mortality, and improved healthcare facilities $[9,13,16]$. As a result of this, the overall population of elderly individuals increased from $7.9 \%$ in 2010 to $10.7 \%$ in 2020 [15]. Therefore, Malaysia is forecasted to become an aging country by 2030, with the percentage of old people rising to about $20 \%$ [14]. This will have a substantial impact on the country's social, cultural, and economic landscapes, as well as its healthcare delivery system $[9,10,12,14,17]$.

Hence, Malaysia must plan to embrace this issue within a shorter time frame compared with other developed countries such as Japan, France, and Germany, who have had decades or even centuries to prepare for the impacts of their ageing population [14]. Consequently, this population group has been designated as one of Malaysia's national health priority groups because they are more likely to develop age-related diseases, have higher morbidity, use health services more frequently, are more likely to develop complications and have a higher demand for specialist services $[14,18]$. They are the biggest group of patients who utilize health care services in Malaysia, accounting for almost $40 \%$ of overall healthcare resources [9,19-21]. Furthermore, they are more likely to be admitted to the hospital and have longer periods of hospitalization $[9,21]$. The elderly are also the most common category of patients that utilize primary care services $[19,20]$.

The elderly is the most prevalent group with limited HL in many countries such as the USA, UK, Germany, Brazil, China, Taiwan, and Vietnam [8,22-26]. The prevalence of limited HL in elderly populations worldwide ranged from 27 to $91.5 \%$ [27-30]. While most of these studies were conducted in a community setting [29-32], one was conducted among patients in a district hospital [28] and another was based on a population registry in Germany [27].

Previous studies have reported several factors that were associated with HL levels among the elderly population. These include education level, ethnicity, income, age, cognitive status, presence of chronic diseases, information seeking through television or internet, and physical performance [4,23,27-30,32-35]. Studies in China, Israel, and Germany reported that elderly persons with no or a low education had lower HL levels $[27-30,33,34]$. A Chinese study found that being in an ethnic minority group was associated with having limited HL [29]. Income deprivation was also associated with limited HL according to studies conducted in China and Germany [27,29,30,32]. Several studies also concluded that older age was linked to limited HL $[23,28,32]$. In contrast, the elderly in Finland and China with a lesser number of chronic diseases had lower levels of limited HL [4,29]. Few studies found that the elderly who sought information through television or the internet were associated with higher HL levels $[23,29,35]$. Finally, Eronen et al. reported that improved cognitive status was associated with higher HL levels among elderly Finns [4].

There are a lack of published data on HL among the elderly in Malaysia [36]. A recent community study among a group of elderly in an urban neighborhood setting in Shah Alam, Selangor, found that $62.6 \%$ of the elderly population had limited HL levels [31]. 
However, there is no published study on the prevalence of limited HL and its associated factors among elderly patients in a primary care setting in Malaysia. Hence, this study aimed to determine the prevalence of limited HL levels and its associated factors among elderly patients in an urban academic primary care clinic in Selangor, Malaysia.

\section{Materials and Methods}

\subsection{Study Design and Population}

This was a cross-sectional study conducted from January 2020 to January 2021 among elderly patients who attended an urban academic primary care clinic in Malaysia. This primary care clinic is located in the Gombak district, Selangor. Services provided here include walk-in clinics for acute presentations and health screening and appointment-based clinics for chronic disease follow up. The clinic has access to radiology, laboratory, and referral services for other specialties. An average of 80 patients attend this clinic per day. All of the doctors working there are family medicine specialists or postgraduate doctors pursuing a Master's degree in family medicine. The vast majority of the patients reside in the surrounding areas of Selangor and Kuala Lumpur.

The patients were chosen based on the inclusion and exclusion criteria. Elderly patients aged at least 60 years who understood English or the Malay language were included. Meanwhile, the elderly who presented with acute medical conditions such as hypertensive emergency, acute coronary syndrome, or psychiatric emergencies, i.e., acute psychosis or delirium, were excluded.

\subsection{Sample Size Determination}

The sample size was determined using the "Sample size for a Proportion of Descriptive Study" formula based on the main objective of the study. The required sample size was based on the prevalence of limited HL among the elderly in Germany (66.3\%) by Vogt et al. [32]. The confidence interval was taken as $95 \%$. Based on these assumptions, the minimum required sample needed for this study was 344 patients. Taking into account a $20 \%$ non-response and non-eligible rate, this study aimed to approach at least 413 participants.

\subsection{Study Tool}

The study tool consisted of two parts. The first part was the sociodemographic and clinical characteristics. The Elderly Cognitive Assessment Questionnaire (ECAQ) is a measure for assessing cognitive impairment generated from the Mini-Mental State Examination and the Geriatric Mental Status Schedule [37]. ECAQ was developed and validated in Singapore by Kua et al. in 1992, and it shows a sensitivity of 85.3 and specificity of $91.5 \%$ with a positive predictive value of $82.8 \%$ [37]. It is available in three languages: English, Malay, and Chinese [21]. It consists of three sections: memory (three items), orientation (six items), and memory recall (one item). ECAQ is scored as the following: (i) $\geq 7$ for normal, (ii) 5-6 for borderline dementia, and (iii) $\leq 4$ for probable dementia.

The second part was the Health Literacy Survey Questionnaire Short Form 12 questions (HLS-SF12) version. The original HLS-SF12 version and the Malay version were developed and validated in a six Asian country study by Duong et al. [38]. It has good reliability (Cronbach's $\alpha$ of 0.85) [38]. As this short questionnaire was developed based on the longer HLS-EU-Q47 questionnaire, a confirmatory factor analysis (CFA) was used to establish construct validity for the three domains of health care $(\mathrm{HC})$, disease prevention (DP), and health promotion (HP) [38,39]. In the CFA, the Comparative Fit Index (CFI) and the Root Mean Square Error of Approximation (RMSEA) were used as the model fit indices. The CFI value was 0.95 , which indicated an acceptable model fit. The RMSEA value of 0.06 represented a good fit. The three-domain model showed a goodness of fit with $\chi^{2} / \mathrm{df}$ (relative chi-square) of 2.38, AGFI (adjusted goodness-of-fit index) of 0.94, GFI (goodness-of-fit index) of 0.96, IFI (incremental fit index) of 0.95, and NFI (normal fit index) 
of 0.92 [38]. The overall results supported the fitness of the four-factor structure within each of the three domains of the HLS-EU-Q47 [38].

Each item of the HLS-SF12 assesses the perceived difficulties in finding, understanding, judging, and applying health information. It uses a four-point Likert scale with responses of 1 = very difficult, 2 = fairly difficult, 3 = fairly easy, and $4=$ very easy. The HL score is calculated using the following formula: HL score $=(M-1) \times(50 / 3)$, where $M=$ mean, $1=$ the minimal possible value of the mean, $3=$ the range of the mean, and $50=$ the chosen maximum value of the desired scale. HL scores $\leq 33$ were classified as limited HL, while $>33$ were classified as adequate HL [38].

\subsection{Patient Recruitment, Sampling Method, and Data Collection}

Elderly patients who attended an urban academic primary care clinic in Malaysia between January 2020 and January 2021 were recruited. Every alternate elderly patient who registered at the clinic was approached in the assessment room and was invited to partake in the study. Next, they were screened for eligibility according to the inclusion and exclusion criteria. Those who were interested were given a participant information sheet containing information about the study background, purpose, and benefits. Eligible participants who consented were assured of their confidentiality, briefed about the study, and were then given the study questionnaire. Data were collected by a trained research assistant to guarantee a uniform data gathering procedure.

Sociodemographic and clinical characteristics collected included the participants' age, gender, marital status, ethnicity, education level, occupation, household income, number of children, living arrangement, ECAQ score, smoking status, alcohol consumption, presence and number of medical illness, family history of medical illness, family members with medical training, perceived health status, accompanied by family to the clinic, finding medical information on the television or internet, and limitations on activities. HL score was determined using the HLS-SF12 questionnaire. The flowchart of this study is shown in Figure 1.

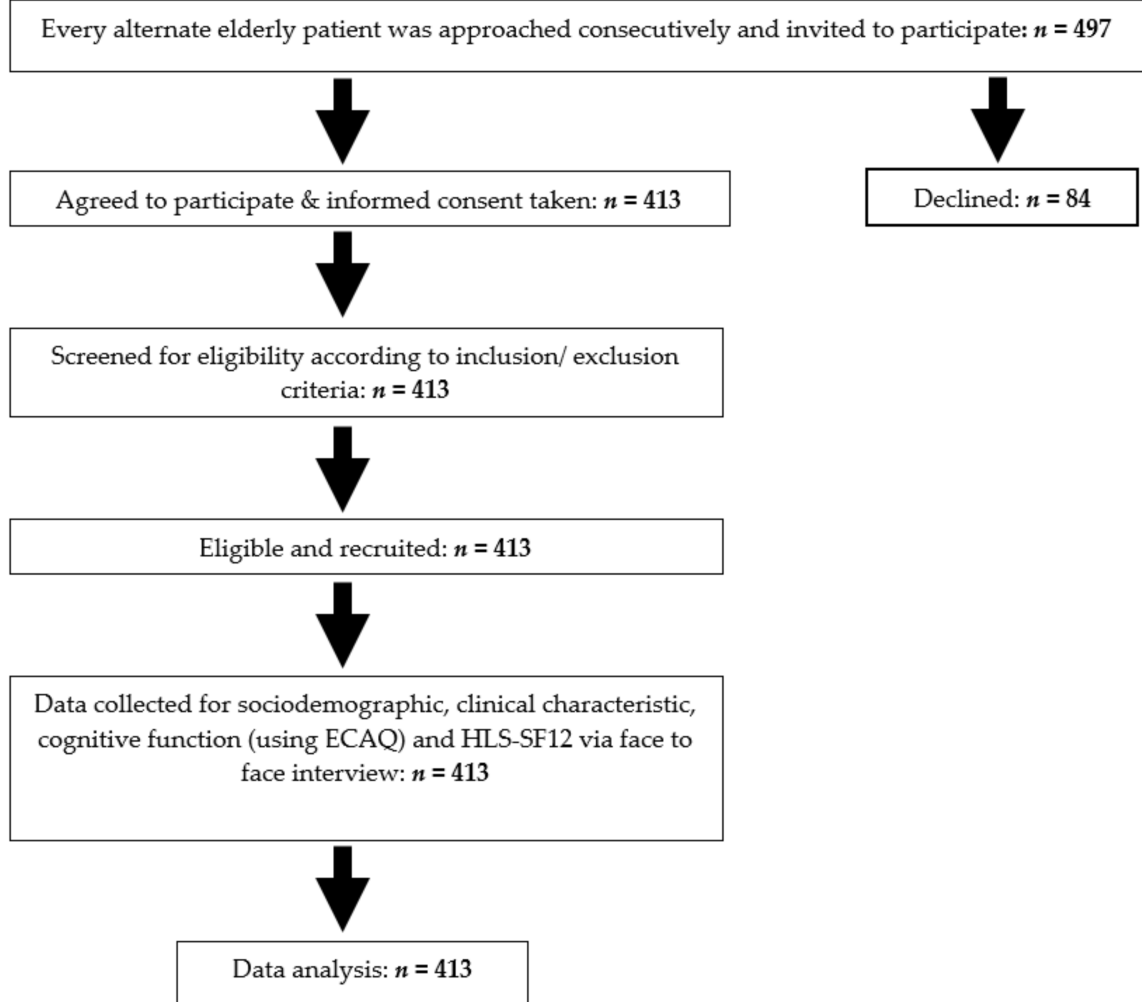

Figure 1. Flow chart of the study. 


\subsection{Definition of Terms}

Regarding the sociodemographic variables, the elderly were divided into three age groups. These are the young-old (60-69 years old), middle-old (70-79 years old), and very-old (over 80 years old) [16,32,40]. Education levels were classified according to the Malaysian education system, as follows: no formal education, primary school (standard 1-6; ages 7-12), secondary school (form 1-5; ages 13-17), and tertiary education (college or university). Household incomes were categorized into two groups: low (B40) with a monthly household income of RM $<4850$, and middle (M40) and high (T20) with a monthly household income of $\mathrm{RM} \geq 4850$ [41].

\subsection{Data Entry and Statistical Analysis}

Data entry and statistical analysis were performed using the IBM ${ }^{\circledR}$ Statistical Package for Social Sciences (SPSS) version 27 software (IBM Corp., Armonk, NY, USA) [42]. Descriptive analysis was used to describe the sociodemographic characteristics, clinical characteristics, and prevalence of limited HL levels of the participants. To describe the continuous data, the mean with standard deviations (SD) and median with interquartile ranges (IQR) were used for normally distributed and non-normally distributed data, respectively. Frequencies and percentages were used to describe the categorical data.

Inferential analysis was conducted to determine the factors associated with limited HL levels. Odds ratios (OR) and their $95 \%$ confidence intervals (CI) were calculated using simple logistic regression (SLogR) and multiple logistic regression (MLogR). Variables with a $p$-value of $<0.05$ from the SLogR were subsequently included in the MLogR. The MLogR was performed using the forward binary logistic regression method. Model fitness was checked using the Hosmer-Lemeshow goodness-of-fit test. Interactions, multicollinearity, and assumptions were also checked. Statistical significance was taken at a $p$-value of $<0.05$.

\subsection{Ethical Consideration}

This study was conducted in compliance with the Declaration of Helsinki and the code of ethics of the World Medical Association [43]. Ethical approval was obtained from the Research Ethics Committee of Universiti Teknologi MARA (600-TNCPI (5/1/6)) (REC/678/19) before the study commenced.

\section{Results}

\subsection{Characteristics of Respondents}

A total of 497 potential participants were approached at the academic primary care clinic. They were selected based on the inclusion and exclusion criteria. Eighty-four patients declined to participate in the study. Therefore, the final number of participants was 413 . The response rate was $83.1 \%$. Table 1 shows the sociodemographic and clinical characteristics of the respondents, stratified by their health literacy levels (limited and adequate). The median age (IQR) was 67 (8). The majority of the participants were 60-69 years old (68.3\%), male (59.8\%), and Malay (85.7\%). Most of the respondents were married $(86 \%)$, had at least a secondary school education (87.2\%), and had normal ECAQ scores (97.3\%). Regarding household income, $77 \%$ of the respondents were from the low-income group (B40). The median HL score and IQR was 41.7 (13.9). Out of 413 respondents, 79 (19.1\% (95\% CI: 15.3, 23)) had limited HL while 334 (80.9\% (95\% CI: 77, 84.7)) had adequate HL. 
Table 1. Sociodemographic and clinical characteristics of the overall participants and according to health literacy level $(N=413)$.

\begin{tabular}{|c|c|c|c|}
\hline \multirow{4}{*}{ Characteristics } & \multicolumn{2}{|c|}{ Health Literacy Level * } & \multirow{2}{*}{ Total } \\
\hline & Limited & Adequate & \\
\hline & $N=79$ & $N=334$ & $N=413$ \\
\hline & $n(\%)$ & $n(\%)$ & $n(\%) * *$ \\
\hline \multicolumn{4}{|l|}{ Health literacy level } \\
\hline Limited & $79(100 \%)$ & - & $79(19.1 \%)$ \\
\hline Adequate & - & $344(100 \%)$ & $334(80.9 \%)$ \\
\hline Age (years): Median [IQR] & & & $67(8)$ \\
\hline \multicolumn{4}{|l|}{ Age Group: $n(\%)$ years } \\
\hline Young-Old Age (60-69) & $29(36.7 \%)$ & $253(75.7 \%)$ & $282(68.3 \%)$ \\
\hline Middle-Old Age (70-79) & $42(53.2 \%)$ & $75(22.5 \%)$ & $117(28.3 \%)$ \\
\hline Very-Old $(\geq 80)$ & $8(10.1 \%)$ & $6(1.8 \%)$ & $14(3.4 \%)$ \\
\hline \multicolumn{4}{|l|}{ Gender: $n(\%)$} \\
\hline Male & $42(53.2 \%)$ & $205(61.4 \%)$ & $247(59.8 \%)$ \\
\hline Female & $37(46.8 \%)$ & $129(38.6 \%)$ & $166(40.2 \%)$ \\
\hline \multicolumn{4}{|l|}{ Ethnicity: $n(\%)$} \\
\hline Malay & $66(83.5 \%)$ & $288(86.2 \%)$ & $354(85.7 \%)$ \\
\hline Others & $13(16.5 \%)$ & $46(13.8 \%)$ & $59(14.3 \%)$ \\
\hline \multicolumn{4}{|l|}{ Marital status: $n(\%)$} \\
\hline Single, widow, and divorcee & $22(27.8 \%)$ & $36(10.8 \%)$ & $58(14 \%)$ \\
\hline Married & $57(72.2 \%)$ & $298(89.2 \%)$ & $355(86 \%)$ \\
\hline \multicolumn{4}{|l|}{ Education level: $n(\%)$} \\
\hline No formal education and primary education & $37(46.8 \%)$ & $16(4.8 \%)$ & $53(12.8 \%)$ \\
\hline Secondary and tertiary & $42(53.2 \%)$ & $318(95.2 \%)$ & $360(87.2 \%)$ \\
\hline \multicolumn{4}{|l|}{ Household income: $n(\%)$} \\
\hline $\mathrm{B} 40(\mathrm{RM}<4850)$ & $66(83.5 \%)$ & $252(75.4 \%)$ & $318(77 \%)$ \\
\hline $\mathrm{M} 40 / \mathrm{T} 20(\mathrm{RM} \geq 4850)$ & $13(16.5 \%)$ & $82(24.6 \%)$ & $95(23 \%)$ \\
\hline \multicolumn{4}{|l|}{ Occupation: $n(\%)$} \\
\hline Employed & $8(10.1 \%)$ & $32(9.6 \%)$ & $40(9.7 \%)$ \\
\hline Retired/Pensioner & $42(53.2 \%)$ & $269(80.5 \%)$ & $311(75.3 \%)$ \\
\hline Unemployed & $29(36.7 \%)$ & $33(9.9 \%)$ & $62(15.0 \%)$ \\
\hline \multicolumn{4}{|l|}{ Children: $n(\%)$} \\
\hline No & $0(0.0 \%)$ & $11(3.3 \%)$ & $11(2.7 \%)$ \\
\hline Yes & $79(100.0 \%)$ & $323(96.7 \%)$ & $402(97.3 \%)$ \\
\hline \multicolumn{4}{|l|}{ Living arrangement: $n(\%)$} \\
\hline Stays alone & $1(1.3 \%)$ & $7(2.1 \%)$ & $8(1.9 \%)$ \\
\hline Stays with family members & $78(98.7 \%)$ & $327(97.9 \%)$ & $405(98.1 \%)$ \\
\hline \multicolumn{4}{|l|}{ Smoking status: $n(\%)$} \\
\hline Non-smoker & $63(79.7 \%)$ & $223(66.8 \%)$ & $286(69.2 \%)$ \\
\hline Former smoker & $14(17.7 \%)$ & $80(24 \%)$ & $94(22.8 \%)$ \\
\hline Smoker & $2(2.5 \%)$ & $31(9.3 \%)$ & $33(8 \%)$ \\
\hline \multicolumn{4}{|l|}{ Alcohol consumption: $n(\%)$} \\
\hline No & $77(97.5 \%)$ & $315(94.3 \%)$ & $392(94.9 \%)$ \\
\hline Yes & $2(6.1 \%)$ & $19(5 \%)$ & $21(5.1 \%)$ \\
\hline \multicolumn{4}{|l|}{$\begin{array}{l}\text { Elderly Cognitive Assessment Questionnaire (ECAQ) } \\
\text { score: } n(\%)\end{array}$} \\
\hline 7-10: Normal & $69(87.3 \%)$ & $333(99.7 \%)$ & $402(97.3 \%)$ \\
\hline$<6$ : Borderline/Probable & $10(12.7 \%)$ & $1(0.3 \%)$ & $11(2.7 \%)$ \\
\hline
\end{tabular}


Table 1. Cont.

\begin{tabular}{|c|c|c|c|}
\hline \multirow{4}{*}{ Characteristics } & \multicolumn{2}{|c|}{ Health Literacy Level * } & \multirow{2}{*}{ Total } \\
\hline & Limited & Adequate & \\
\hline & $N=79$ & $N=334$ & $N=413$ \\
\hline & $n(\%)$ & $n(\%)$ & $n(\%) * *$ \\
\hline \multicolumn{4}{|l|}{ Medical illness: $n(\%)$} \\
\hline No & $2(2.5 \%)$ & $20(6 \%)$ & $22(5.3 \%)$ \\
\hline Yes & $77(97.5 \%)$ & $314(94 \%)$ & $391(94.7 \%)$ \\
\hline \multicolumn{4}{|c|}{ Number of medical illnesses: $n(\%)(n=391)$} \\
\hline 1 & $11(14.3 \%)$ & $59(18.8 \%)$ & $70(17.9 \%)$ \\
\hline 2 & $15(19.5 \%)$ & $96(30.6 \%)$ & $111(28.4 \%)$ \\
\hline$\geq 3$ & $51(66.2 \%)$ & $159(50.6 \%)$ & $210(53.7 \%)$ \\
\hline \multicolumn{4}{|c|}{ Family History of Medical Illness: $n(\%)$} \\
\hline No & $30(38 \%)$ & $72(21.6 \%)$ & $102(24.7 \%)$ \\
\hline Yes & $49(62 \%)$ & $262(78.4 \%)$ & $311(75.3 \%)$ \\
\hline \multicolumn{4}{|c|}{ Family members with medical training: $n(\%)$} \\
\hline No & $56(70.9 \%)$ & $247(74 \%)$ & $303(73.4 \%)$ \\
\hline Yes & $23(29.1 \%)$ & $87(26 \%)$ & $110(26.6 \%)$ \\
\hline \multicolumn{4}{|c|}{ Perceived health status: $n(\%)$} \\
\hline Very poor/poor & $6(7.6 \%)$ & $24(7.2 \%)$ & $30(7.3 \%)$ \\
\hline Fair & $32(40.5 \%)$ & $118(35.3 \%)$ & $150(36.3 \%)$ \\
\hline Good & $37(46.8 \%)$ & $160(47.9 \%)$ & $197(47.7 \%)$ \\
\hline Very Good & $4(5.1 \%)$ & $32(9.6 \%)$ & $36(8.7 \%)$ \\
\hline \multicolumn{4}{|c|}{ Accompanied by family to the clinic: $n(\%)$} \\
\hline No & $21(26.6 \%)$ & $150(44.9 \%)$ & $171(41.4 \%)$ \\
\hline Yes & $58(73.4 \%)$ & $184(55.1 \%)$ & $242(58.6 \%)$ \\
\hline \multicolumn{4}{|c|}{$\begin{array}{l}\text { Finding medical information on television (TV) or the } \\
\text { internet: } n(\%)\end{array}$} \\
\hline No & $36(45.6 \%)$ & $48(14.4 \%)$ & $84(20.3 \%)$ \\
\hline Yes & $43(54.4 \%)$ & $286(85.6 \%)$ & $329(79.7 \%)$ \\
\hline \multicolumn{4}{|c|}{ Limitations on activities: $n(\%)$} \\
\hline No & $59(74.4 \%)$ & $271(81.1 \%)$ & 330 (79.9\%) \\
\hline Yes & $20(25.3 \%)$ & $63(18.9 \%)$ & $83(20.1 \%)$ \\
\hline
\end{tabular}

* Data presented as row percentage. ${ }^{* *}$ Data presented as column percentage. IQR-interquartile range.

\subsection{Factors Associated with Limited $H L$}

Table 2 shows the results of the univariate analysis using Simple Logistic Regression. From the univariate analysis, age, marital status, education, occupation, number of children, ECAQ score, smoking status, family history of medical illness, finding medical information through the television or internet, and being accompanied to the clinic had a significant $p$-value of $<0.05$.

Table 2. Simple logistic regressions on the factors associated with limited health literacy level among elderly patients.

\begin{tabular}{|c|c|c|c|c|}
\hline Characteristics & Beta (SE) & Wald (df) & OR $(95 \% \mathrm{Cl})$ & $p$-Value \\
\hline \multicolumn{5}{|l|}{ Age Group } \\
\hline Young-Old (60-69) & & & 1 & ref \\
\hline Middle-Old (70-79) & $1.59(0.28)$ & $33.29(1)$ & $4.89(2.85,8.37)$ & $<0.001$ * \\
\hline Very-Old $(\geq 80)$ & $2.45(0.58)$ & $18.24(1)$ & $11.63(3.77,35.87)$ & $<0.001 *$ \\
\hline \multicolumn{5}{|l|}{ Gender } \\
\hline Male & & & 1 & ref \\
\hline Female & $0.34(0.25)$ & $1.78(1)$ & $1.40(0.85,2.29)$ & 0.182 \\
\hline \multicolumn{5}{|l|}{ Ethnicity } \\
\hline Malay & & & 1 & ref \\
\hline Others & $0.21(0.34)$ & $0.38(1)$ & $1.23(0.63,2.41)$ & 0.54 \\
\hline
\end{tabular}


Table 2. Cont.

\begin{tabular}{|c|c|c|c|c|}
\hline Characteristics & Beta (SE) & Wald (df) & OR $(95 \% \mathrm{Cl})$ & $p$-Value \\
\hline $\begin{array}{l}\text { Marital status } \\
\text { Single/Widow/Divorce } \\
\text { Married }\end{array}$ & $-1.16(0.31)$ & $14.33(1)$ & $\begin{array}{c}1 \\
0.31(0.17,0.57)\end{array}$ & $\begin{array}{c}\text { ref } \\
<0.001 *\end{array}$ \\
\hline $\begin{array}{l}\text { Education level } \\
\text { No formal/primary } \\
\text { Secondary/tertiary }\end{array}$ & $-2.86(0.34)$ & $70.36(1)$ & $\begin{array}{c}1 \\
0.06(0.03,0.11)\end{array}$ & $\begin{array}{c}\text { ref } \\
<0.001 *\end{array}$ \\
\hline $\begin{array}{c}\text { Household income } \\
\text { B40 }(\mathrm{RM}<4850) \\
\mathrm{M} 40 / \mathrm{T} 20(\mathrm{RM} \geq 4850)\end{array}$ & $-0.50(0.33)$ & $2.33(1)$ & $\begin{array}{c}1 \\
0.61(0.32,1.15) \\
\end{array}$ & $\begin{array}{c}\text { ref } \\
0.127\end{array}$ \\
\hline $\begin{array}{l}\text { Occupation } \\
\text { Unemployed } \\
\text { Employed } \\
\text { Retired/Pensioner }\end{array}$ & $\begin{array}{l}-1.26(0.47) \\
-1.73(0.30)\end{array}$ & $\begin{array}{c}7.15(1) \\
32.34(1)\end{array}$ & $\begin{array}{c}1 \\
0.28(0.11,0.72) \\
0.18(0.10,0.32)\end{array}$ & $\begin{array}{l}\text { ref } \\
0.007 * \\
<0.001 *\end{array}$ \\
\hline $\begin{array}{c}\text { Number of children } \\
\qquad \begin{array}{c}1-2 \\
\geq 3\end{array}\end{array}$ & $1(0.45)$ & $4.98(1)$ & $\begin{array}{c}1 \\
2.72(1.13,6.55)\end{array}$ & $\begin{array}{c}\text { ref } \\
0.026^{*}\end{array}$ \\
\hline $\begin{array}{c}\text { Living arrangement } \\
\text { Stays alone } \\
\text { Stays with family members }\end{array}$ & $0.51(1.08)$ & $0.23(1)$ & $\begin{array}{c}1 \\
1.67(0.20,13.77)\end{array}$ & $\begin{array}{c}\text { ref } \\
0.634\end{array}$ \\
\hline $\begin{array}{l}\text { Smoking status } \\
\text { Non-smoker } \\
\text { Former smoker } \\
\text { Smoker }\end{array}$ & $\begin{array}{l}-0.48(0.32) \\
-1.48(0.74)\end{array}$ & $\begin{array}{l}2.20(1) \\
3.95(1)\end{array}$ & $\begin{array}{c}1 \\
0.62(0.33,1.17) \\
0.23(0.05,0.98)\end{array}$ & $\begin{array}{c}\text { ref } \\
0.138 \\
0.047^{*}\end{array}$ \\
\hline $\begin{array}{l}\text { Alcohol consumption } \\
\text { No } \\
\text { Yes }\end{array}$ & $-0.84(0.75)$ & $1.25(1)$ & $\begin{array}{c}1 \\
0.43(0.10,1.89)\end{array}$ & $\begin{array}{c}\text { ref } \\
0.264\end{array}$ \\
\hline $\begin{array}{c}\text { Elderly Cognitive Assessment } \\
\text { Questionnaire (ECAQ) } \\
\text { Normal }(7-10) \\
\text { Borderline/Probable dementia }(\leq 6)\end{array}$ & $3.88(1.06)$ & $13.45(1)$ & $\begin{array}{c}1 \\
48.26(6.08,383.19)\end{array}$ & $\begin{array}{c}\text { ref } \\
<0.001^{*}\end{array}$ \\
\hline $\begin{array}{l}\text { Medical illness } \\
\text { No } \\
\text { Yes }\end{array}$ & $0.90(0.75)$ & $1.42(1)$ & $\begin{array}{c}1 \\
2.45(0.56,10.72)\end{array}$ & $\begin{array}{c}\text { ref } \\
0.233\end{array}$ \\
\hline $\begin{array}{c}\text { Number of medical illness } \\
1 \\
2 \\
\geq 3\end{array}$ & $\begin{array}{c}-0.18(0.43) \\
0.54(0.37)\end{array}$ & $\begin{array}{l}0.17(1) \\
2.20(1) \\
\end{array}$ & $\begin{array}{c}1 \\
0.84(0.36,1.95) \\
1.72(0.84,3.52)\end{array}$ & $\begin{array}{c}\text { ref } \\
0.681 \\
0.138\end{array}$ \\
\hline $\begin{array}{c}\text { Family History of Medical Illness } \\
\text { No } \\
\text { Yes }\end{array}$ & $-0.80(0.27)$ & $8.98(1)$ & $\begin{array}{c}1 \\
0.45(0.27,0.76)\end{array}$ & $\begin{array}{c}\text { ref } \\
0.003 *\end{array}$ \\
\hline $\begin{array}{c}\text { Family member with medical training } \\
\text { No } \\
\text { Yes }\end{array}$ & $0.15(0.28)$ & $0.31(1)$ & $\begin{array}{c}1 \\
1.17(0.68,2.01)\end{array}$ & $\begin{array}{c}\text { ref } \\
0.58\end{array}$ \\
\hline $\begin{array}{c}\text { Perceived health status } \\
\text { Very poor/poor } \\
\text { Fair } \\
\text { Good } \\
\text { Very Good }\end{array}$ & $\begin{array}{l}0.08(0.50) \\
-0.08(0.49) \\
-0.69(0.70)\end{array}$ & $\begin{array}{l}0.03(1) \\
0.03(1) \\
0.98(1)\end{array}$ & $\begin{array}{c}1 \\
1.09(0.41,2.88) \\
0.93(0.35,2.42) \\
0.50(0.13,1.97)\end{array}$ & $\begin{array}{c}\text { ref } \\
0.87 \\
0.874 \\
0.322\end{array}$ \\
\hline $\begin{array}{c}\text { Accompanied by family to the clinic } \\
\text { No } \\
\text { Yes }\end{array}$ & $0.81(0.28)$ & $8.56(1)$ & $\begin{array}{c}1 \\
2.25(1.31,3.88)\end{array}$ & $\begin{array}{c}\text { ref } \\
0.003 *\end{array}$ \\
\hline
\end{tabular}


Table 2. Cont

\begin{tabular}{|c|c|c|c|c|}
\hline Characteristics & Beta (SE) & Wald (df) & OR $(95 \% \mathrm{Cl})$ & $p$-Value \\
\hline \multicolumn{5}{|c|}{$\begin{array}{l}\text { Finding medical information on television } \\
\qquad(\mathrm{TV}) \text { or the internet }\end{array}$} \\
\hline No & & & 1 & ref \\
\hline Yes & $-1.61(0.28)$ & $34.27(1)$ & $0.20(0.12,0.34)$ & $<0.001 *$ \\
\hline \multicolumn{5}{|c|}{ Limitations on activities } \\
\hline No & & & 1 & ref \\
\hline Yes & $0.38(0.29)$ & $1.64(1)$ & $1.46(0.82,2.60)$ & 0.2 \\
\hline
\end{tabular}

* Statistically significant at $p<0.05$; Statistical test—Simple Logistic Regression; OR—odds ratio; CI—confidence interval; ref-reference group.

These 12 variables were included in the Multiple Logistic Regression (MLogR) model. Table 3 shows the factors associated with limited HL among elderly patients, with $p$-values $<0.05$. The middle-old age (70-79 years old; Adj OR 4.05, 95\% CI 2.19, 7.52) and very-old age groups ( $\geq 80$ years old; Adj OR 4.36, 95\% CI 1.02, 18.63) were four times more likely to have limited HL levels compared with the young-old age group. Compared with those who did not, those who found medical information on the television/internet have lower odds of having limited HL (Adj OR 0.21, 95\% CI 0.05, 0.93). Furthermore, patients who had at least a secondary school education (Adj OR 0.06, 95\% CI 0.02, 0.24) had a lower risk of limited HL than those with non or a primary education. The $\mathrm{R}^{2}$ value was 0.25 , which denotes $25 \%$ of the associated factors contributed to the limited HL among our study population.

Table 3. Multiple logistic regressions on the factors associated with a limited health literacy level among elderly patients.

\begin{tabular}{|c|c|c|c|c|}
\hline Characteristics & Adjusted Beta (SE) & Wald (df) & Adjusted OR (95\% CI) & $p$-Value \\
\hline \multicolumn{5}{|l|}{ Age Group years } \\
\hline Young-Old (60-69) & & & 1 & ref \\
\hline Middle-Old (70-79) & $1.40(0.32)$ & $19.7(1)$ & $4.05(2.19,7.52)$ & $<0.001$ * \\
\hline Very-Old $(\geq 80)$ & $1.47(0.74)$ & $3.94(1)$ & $4.36(1.02,18.63)$ & 0.047 * \\
\hline \multicolumn{5}{|c|}{$\begin{array}{l}\text { Finding medical information on television } \\
\text { (TV) or the internet }\end{array}$} \\
\hline No & & & 1 & ref \\
\hline Yes & $-1.55(0.75)$ & $4.26(1)$ & $0.21(0.05,0.93)$ & $<0.001$ * \\
\hline \multicolumn{5}{|l|}{ Education } \\
\hline No formal/Primary education & & & 1 & ref \\
\hline Secondary/Tertiary & $-2.83(0.71)$ & $15.82(1)$ & $0.06(0.02,0.24)$ & $<0.001 *$ \\
\hline
\end{tabular}

The model reasonably fits well (Hosmer-Lemeshow test: $p=0.822$ ); model assumptions were met; no significant interactions and multicollinearity problem; model explained between $24.7 \%$ (Cox and Snell $\mathrm{R}^{2}$ ) and $39.7 \%$ (Nagelkerke $\mathrm{R}^{2}$ ) of the variance in limited HL and correctly classified $87.2 \%$ of cases; sensitivity $40.5 \%$, specificity $98.2 \%$. ${ }^{*}$ Statistical significance at $p<0.05$; OR—odds ratio; CI—confidence interval; $\mathrm{df}$ - degree of freedom; ref-reference group.

\section{Discussion}

To the best of our knowledge, this is one of the few studies conducted in a developing country to determine HL levels among elderly patients attending a primary care clinic. This study is in line with international research that recognizes the elderly as a critical population for HL research $[18,20]$. Furthermore, the majority of published studies on HL do not focus on elderly patients and the factors that influence them in primary care settings.

This study discovered that the prevalence of limited HL in our study population was $19.1 \%$. This is significantly lower compared with a study among the elderly in the community of Shah Alam, Selangor, Malaysia, which reported a prevalence of $62.6 \%$ [31]. Comparable to our finding, Tiller et al. reported that the prevalence of limited HL among the German elderly in an urban setting was $27 \%$ [27]. However, studies from other coun- 
tries have reported a significantly higher prevalence of limited HL among their elderly populations-Taiwan (57.6\%), Germany $(66.3 \%)$, and China (91.5\%) $[28,30,32]$. Compared with all of these countries, the prevalence of limited HL was the lowest in our study. One of the reasons for this could be that our population involved patients who had chronic diseases and would have had regular access to health-care services provided by clinicians who are skilled in their field [20,44,45]. All of the doctors at this clinic were either family medicine specialists or postgraduate doctors pursuing a Master's degree in family medicine. The ongoing clinical training and continuous professional development activities they undergo may have an effect on these patients' HL levels [20,45,46].

We also found that in comparison with the young-old age group, the middle-old and very-old age groups were four times more likely to have limited HL. These findings are consistent with studies conducted in the United States of America, the United Kingdom, Germany, Brazil, China, Taiwan, and Vietnam, which found that older persons were more likely to have limited HL [8,22-26,32]. Elderly patients may process information at a slower pace and have less working memory (the ability to process multiple bits of information at the same time) $[47,48]$. Their reduced cognitive abilities may limit their ability to participate and comprehend the health information they receive [47,48]. In contrast, Nakayama et al.'s study in Japan found that HL increased with age [49]. They proposed that this could be due to factors involving interactions between different age populations, educational background, and active internet usage within the Japanese population [49].

Education is also an important factor for HL. From our findings, those with secondary or tertiary education levels had lower odds of having limited HL than those with lower education levels. The relationship between higher education levels and increased HL levels were also reported in recent studies among the elderly in Germany, Israel, Taiwan, and China $[27-29,33,34,50,51]$. Similar findings were reported in previous studies conducted in Malaysia among type 2 diabetes mellitus patients by Esahak et al., and among overweight housewives by Shahrir et al. [50,51]. Those with higher education levels presumably have better knowledge to empower themselves to take control of their health care and subsequently increase their HL level [50]. It is presumed that people with a higher level of education are more likely to have health-seeking behaviors and critical thinking, which drives them to obtain more health-related information and increase their HL level [50]. This is a predicted outcome because older individuals, especially in developing countries, are more likely to have limited access to education, especially in impoverished countries $[17,23,28]$. An example is Taiwan, where many adults over the age of 85 are illiterate and have never attended school because schooling was not made compulsory to them [28]. However, several studies observed that there is no link between education level and HL level $[48,49]$. Nielsen-Bohlman and colleagues suggested that even persons with high literacy skills may struggle to understand health information if they lack the knowledge to understand the medical jargon used and the context of the interactions with healthcare practitioners [2].

In this digital age, technology has become one of the most important components to empower people to take control of their health. Consistent with this, our study found a significant association between lower risks of limited HL and finding medical knowledge via the television and internet. Therefore, the elderly can enhance their HL and be motivated to learn more by using the internet or television. Television, according to Li et al., can be used to assist individuals to learn more about their health [29]. Those who received health information through television had a better probability of improving their HL [29]. Van Hoa et al. also found that elderly people in Vietnam who used the internet frequently had a higher level of HL [23]. The internet is a valuable resource to find information. According to a study by Levy et al., lack of internet usage is a factor that contributed to having a limited HL level [35]. In Malaysia, Shahrir et al. found that lack of internet connectivity was linked to having limited HL levels [51]. The accessibility to health information via the mass media and internet is linked to health behavior, information seeking, and ultimately health outcomes of patients [52-54]. This health information accessibility has become one 
of the most important factors impacting the HL level. The digital platform provides people with health information to self-evaluate and make decisions about their health care, disease prevention, and health promotion $[52,54]$. However, the general public may need guidance from health practitioners or authorities regarding reputable sources of validated medical information.

\subsection{Strengths and Limitations}

To the best of our knowledge, this is the first study to determine the prevalence of limited HL and its associated factors among elderly patients in a primary care setting in Malaysia. However, this study has several limitations. First, because of the cross-sectional study design, the study findings could only show association but not causality. Other than that, this study was conducted in an urban university primary care setting, where the majority of participants were of Malay ethnicity, and all doctors were either family medicine specialists or postgraduate doctors pursuing a Master's degree in family medicine. Therefore, the findings may not be generalizable to all primary care settings in Malaysia. Finally, several other factors including depression symptoms and health impairments involving vision, hearing, life-space mobility, and physical performance, which may affect HL levels, were not included in this study. Thus, the results from the multiple logistic regression should be interpreted based on the variables included in the regression model.

\subsection{Implications on Clinical Practice and Future Research}

Our study findings have several implications on clinical practice. Firstly, clinics utilizing an electronic medical records (EMR) system can program their system to discreetly "flag" elderly patients aged $\geq 70$ years old, or those with no formal or a low education level. These cues will alert doctors of the possibility that these patients may have limited HL. Doctors can then consciously use communication strategies that may increase these patients' understanding about their medical condition and improve their health literacy. These strategies can be divided into verbal communication, visual aids, and patient selfmanagement and empowerment. Verbal communication strategies include using plain language, limiting information amount and assessing patients' comprehension $[55,56]$. Using visual aids such as simple pictures or videos may also improve patients' understanding of their condition or a medical procedure [55]. Examples of patient self-management and empowerment strategies include encouraging patients to ask two to three questions during their appointments, and assessing patients' understanding of their medications [55].

Secondly, doctors should encourage elderly patients to seek medical information via mass media or the internet. These patients should be guided to reputable sources and websites to get medical information, such as the Ministry of Health Malaysia's MyHealth website. However, to produce effective and easily accessible sources of medical information, collaboration involving the health and information technology, communication, and education sectors need to be strengthened. These contents need to be written at an appropriate reading level (generally 10-11 years old) according to The National Work Group on Literacy and Health [56], using short sentences with minimal medical jargon, and using bulleted lists instead of blocks of text [55]. These contents should also be formatted to suit smartphone interfaces, as most Malaysians access the internet through their smartphones [57].

To facilitate the elderly and the wider population to access health-related information, the Malaysian government should continue to adopt policies to improve the affordability of gadgets to access the internet. In 2013, as part of the National Broadband Initiative (NBI), a one-off rebate of RM200 was given for the purchase of a 3G smartphone, which benefited around 1.5 million youths of Malaysia [58]. Similar initiatives should be offered to the elderly population. According to the latest statistics from the Malaysian Communications and Multimedia Commission (MCMC), mobile broadband subscriptions were ten times higher compared with fixed-broadband subscriptions in Malaysia [59]. However, the Hand Phone Users Survey 2018 reported that network coverage and internet speed were 
still the most highly cited reasons for mobile broadband data user dissatisfaction [60]. Network problems were the main reason for complaints regarding high-speed broadband services [61]. Hence, mobile and fixed broadband internet infrastructures should continue to be improved in order to increase their coverage for the entire country, including rural areas. Reassuringly, actions are underway that are in line with the National Broadband Initiative and National Fiberisation and Connectivity Plan (NFCP) 2019-2023 [58,62]. The NFCP aims to provide affordable and high levels of high quality broadband internet connectivity to the entire country by 2023 [62].

The Ministry of Health should consider establishing policies that promote health literacy in written, multimedia, and internet-based communication directed to the public. According to the World Health Organization's publication titled "Health Literacy: The Solid Facts", the literacy demands of health systems and the literacy skills of average adults are mismatched [63]. To address this, several strategies that can be applied in health care facilities include simplifying the written language used for signages, forms, patient information leaflets, medication instructions, and discharge instructions [63].

Regarding implications for future research, our study findings on the factors that influence older people's HL has created more areas for in-depth research. Future research involving other primary care clinics in Malaysia that include other ethnic groups and rural populations should be performed to confirm the findings of this study. Other factors that may be associated with HL level, including depressive symptoms and health impairments involving vision, hearing, life-space mobility, and physical performance, should be studied. A study assessing HL-related knowledge, attitude, practices, and perceived barriers among primary care doctors in Malaysia is currently underway in order to better understand the other side of the HL interface.

\section{Conclusions}

In conclusion, the prevalence of limited HL levels among the elderly patients in this academic primary care clinic was low. Elderly patients with a good educational background and the ability to find information through the internet or television were found to be associated with lower risks of having limited HL levels, while increased age was associated with higher odds of having limited HL. These factors should be considered in future interventions in order to improve the health of these patients.

Author Contributions: Conceptualization, S.N.H.A.-R., M.-S.M.-Y., S.A.-R. and N.B.; data curation, S.N.H.A.-R., M.-S.M.-Y. and M.R.I.; formal analysis, S.N.H.A.-R. and M.R.I.; investigation, S.N.H.A.R. and M.-S.M.-Y.; methodology, S.N.H.A.-R., M.-S.M.-Y., S.A.-R. and N.B.; project administration, M.-S.M.-Y., S.A.-R. and N.B.; software, S.N.H.A.-R. and M.R.I.; supervision, M.-S.M.-Y., S.A.-R., M.R.I. and N.B.; visualization, S.N.H.A.-R., M.-S.M.-Y. and M.R.I.; writing-original draft, S.N.H.A.-R.; writing-review and editing, M.-S.M.-Y., S.A.-R., M.R.I. and N.B. All authors have read and agreed to the published version of the manuscript.

Funding: This research received no external funding.

Institutional Review Board Statement: The study was conducted according to the guidelines of the Declaration of Helsinki and was approved by the Research Ethics Committee of Universiti Teknologi MARA (600-IRMI (5/1/6)/REC/61/19) before conducting the study.

Informed Consent Statement: Informed consent was obtained from all subjects involved in the study.

Data Availability Statement: Data are kept at the Department of Primary Care Medicine, Universiti Teknologi MARA, in Selangor, Malaysia. Data will be shared upon request and are subject to the applicable and relevant personal data protection laws and regulations.

Acknowledgments: We would like to thank Tin Tin Su and Asian Health Literacy Association (AHLA) for permitting us to use the HLS-SF12 Malay version. Our appreciation is extended to Kua Ee Heok for the permission granted to use the ECAQ cognitive assessment questionnaire. We would 
also like to extend our thanks to Jazmina Aalya, Siti Zubaidah, all the staff of Clinic 1 UiTM Selayang campus, and all participants who were involved directly or indirectly in this study.

Conflicts of Interest: The authors declare no conflict of interest.

\section{References}

1. World Health Organization. Health Literacy Toolkit for Low and Middle-Income Countries: A Series of Information Sheets to Empower Communities and Strengthen Health Systems; Regional Office for South-East Asia: New Delhi, India, 2015.

2. Institute of Medicine (US) Committee on Health Literacy. Health Literacy: A Prescription to End Confusion; Nielsen-Bohlman, L., Panzer, A.M., Kindig, D.A., Eds.; National Academies Press: Washington, DC, USA, 2004.

3. Chesser, A.K.; Keene Woods, N.; Smothers, K.; Rogers, N. Health literacy and older adults: A systematic review. Gerontol. Geriatr Med. 2016, 2, 2333721416630492. [CrossRef]

4. Eronen, J.; Paakkari, L.; Portegijs, E.; Saajanaho, M.; Rantanen, T. Assessment of health literacy among older Finns. Aging Clin. Exp. Res. 2018, 31, 549-556. [CrossRef]

5. Osborne, R.H.; Batterham, R.W.; Elsworth, G.R.; Hawkins, M.; Buchbinder, R. The grounded psychometric development and initial validation of the Health Literacy Questionnaire (HLQ). BMC Public Health 2013, 13, 658. [CrossRef]

6. Holroyd-Leduc, J.; Resin, J.; Ashley, L.; Barwich, D.; Elliott, J.; Huras, P.; Légaré, F.; Mahoney, M.; Maybee, A.; McNeil, H.; et al. Giving voice to older adults living with frailty and their family caregivers: Engagement of older adults living with frailty in research, health care decision making, and in health policy. Res. Involv. Engag. 2016, 2, 1-19. [CrossRef]

7. Samerski, S. Health literacy as a social practice: Social and empirical dimensions of knowledge on health and healthcare. Soc. Sci. Med. 2019, 226, 1-8. [CrossRef] [PubMed]

8. $\quad$ Baker, D.W.; Wolf, M.S.; Feinglass, J.; Thompson, J.A.; Gazmararian, J.A.; Huang, J. Health literacy and mortality among elderly persons. Arch. Intern. Med. 2007, 167, 1503-1509. [CrossRef] [PubMed]

9. Yunus, N.M.; Manaf, N.A.; Omar, A.; Salleh, M. Determinants of healthcare utilisation among the elderly in Malaysia institutions and economies. Inst. Econ. 2017, 9, 115-140.

10. Rasu, R.S.; Bawa, W.A.; Suminski, R.; Snella, K.; Warady, B. Health literacy impact on national healthcare utilization and expenditure. Int. J. Health Policy Manag. 2015, 4, 747-755. [CrossRef]

11. Christensen, K.; Doblhammer, G.; Rau, R.; Vaupel, J. Ageing populations: The challenges ahead. Lancet 2009, 374, 1196-1208. [CrossRef]

12. World Bank Group. World Bank Support to Aging Countries; World Bank—Independent Evaluation Group: Washington, DC, USA, 2019.

13. Chung, C.; Pazim, K.; Mansur, K. Ageing population: Policies and programmes for older people in Malaysia. Asian J. Educ. Soc. Stud. 2020, 2, 92-96.

14. Institute for Public Health (IPH). National Health and Morbidity Survey 2018 (NHMS 2018): Elderly Health-Methodology and General Findings; Institute for Public Health: Dhaka, Bangladesh, 2018.

15. Department of Statistics Malaysia Official Website-Current Population Estimates, Malaysia. 2020. Available online: https: / / www.dosm.gov.my (accessed on 3 July 2021).

16. United Nations Population Fund (UNFPA); HelpAge International. Ageing in the Twenty-First Century: A Celebration and A Challenge; UNFPA: New York, NY, USA; HelpAge International: London, UK, 2012.

17. Ahmad, W.I.W.; Ismail, Z. Some demographic aspects of population aging in Malaysia. World Appl. Sci. J. 2014, 7, 891-894.

18. Yunus, M.M.; Manaf, N.H.A.; Omar, A.; Juhdi, N. Healthcare utilisation among elderly in Malaysia: The mediating role of health literacy. Med. J. Malays. 2021, 76, 218-222.

19. Poi, P.J.-H.; Forsyth, D.R.; Chan, D.K.Y. Services for older people in Malaysia: Issues and challenges. Age Ageing 2004, 33, 444-446. [CrossRef] [PubMed]

20. Ambigga, K.S.; Ramli, A.S.; Suthahar, A.; Tauhid, N.; Clearihan, L.; Browning, C. Bridging the gap in ageing: Translating policies into practice in Malaysian primary care. Asia Pac. Fam. Med. 2011, 10, 2. [CrossRef]

21. Institute for Public Health (Malaysia). National Health and Morbidity Survey (NHMS). Non-Communicable Diseases, Healthcare Demand, and Health Literacy —Key Findings; Institute for Public Health: Putrajaya, Malaysia, 2019.

22. Hagger, M.S.; Hardcastle, S.J.; Hu, M.; Kwok, S.; Lin, J.; Nawawi, H.; Pang, J.; Santos, R.D.; Soran, H.; Su, T.-C.; et al. Health literacy in familial hypercholesterolemia: A cross-national study. Eur. J. Prev. Cardiol. 2018, 25, 936-943. [CrossRef]

23. Van Hoa, H.; Giang, H.T.; Vu, P.T.; Van Tuyen, D.; Khue, P.M. Factors associated with health literacy among the elderly people in Vietnam. Biomed. Res. Int. 2020, 2020, 3490635. [CrossRef]

24. Protheroe, J.; Whittle, R.; Bartlam, B.; Estacio, E.V.; Clark, L.; Kurth, J. Health literacy, associated lifestyle and demographic factors in adult population of an English city: A cross-sectional survey. Health Expect. 2016, 20, 112-119. [CrossRef]

25. Sørensen, K.; Pelikan, J.M.; Röthlin, F.; Ganahl, K.; Slonska, Z.; Doyle, G.; Fullam, J.; Kondilis, B.; Agrafiotis, D.; Uiters, E.; et al Health literacy in Europe: Comparative results of the European Health Literacy Survey (HLS-EU). Eur. J. Public Health 2015, 25, 1053-1058. [CrossRef] [PubMed]

26. Duong, V.T.; Lin, I.F.; Sorensen, K.; Pelikan, J.M.; Van den Broucke, S.; Lin, Y.C.; Chang, P.W. Health literacy in Taiwan: A population-based study. Asia Pac. J. Public Health 2015, 27, 871-880. [CrossRef] 
27. Tiller, D.; Herzog, B.; Kluttig, A.; Haerting, J. Health literacy in an urban elderly East-German population-Results from the population-based CARLA study. BMC Public Health 2015, 15, 1-9. [CrossRef]

28. Chiu, H.-T.; Tsai, H.-W.; Kuo, K.; Leung, A.; Chang, Y.-M.; Lee, P.-H.; Hou, W.-H. Exploring the influencing factors of health literacy among older adults: A cross-sectional survey. Medicina 2020, 56, 330. [CrossRef] [PubMed]

29. Li, C.; Guo, Y. The effect of socio-economic status on health information literacy among urban older adults: Evidence from Western China. Int. J. Environ. Res. Public Health 2021, 18, 3501. [CrossRef]

30. Ma, T.; Meng, H.; Ye, Z.; Jia, C.; Sun, M.; Liu, D. Health literacy mediates the association between socioeconomic status and productive aging among elderly Chinese adults in a newly urbanized community. Front. Public Health 2021, 9. [CrossRef] [PubMed]

31. Yunus, R.M.; Saman, M.S.A.; Zubillah, A.; Juni, K.B.; Gaairibi, A.S.A.; Yahaya, A.N. Health literacy among urban Malaysian elders: A descriptive study. ASM Sc. J. 2020, 13, 7-12.

32. Vogt, D.; Schaeffer, D.; Messer, M.; Berens, E.-M.; Hurrelmann, K. Health literacy in old age: Results of a German cross-sectional study. Health Promot. Int. 2017, 33, 739-747. [CrossRef]

33. Qin, $\mathrm{L} . ; \mathrm{Xu}, \mathrm{H}$. A cross-sectional study of the effect of health literacy on diabetes prevention and control among elderly individuals with prediabetes in rural China. BMJ Open 2016, 6, e011077. [CrossRef]

34. Hochhauser, M.; Brusovansky, M.; Sirotin, M.; Bronfman, K. Health literacy in an Israeli elderly population. Isr. J. Health Policy Res. 2019, 8, 1-9. [CrossRef]

35. Levy, H.; Janke, A.T.; Langa, K. Health literacy and the digital divide among older Americans. J. Gen. Intern. Med. 2014, 30, 284-289. [CrossRef] [PubMed]

36. Abdullah, A.; Liew, S.M.; Salim, H.S.; Ng, C.J.; Chinna, K. Health literacy research in Malaysia: A scoping review. Sains Malays. 2020, 49, 1021-1036. [CrossRef]

37. Kua, E.H.; Ko, S.M. A questionnaire to screen for cognitive impairment among elderly people in developing countries. Acta Psychiatr. Scand. 1992, 85, 119-122. [CrossRef]

38. Duong, T.V.; Aringazina, A.; Kayupova, G.; Nurjanah; Pham, T.V.; Pham, K.M.; Truong, T.Q.; Nguyen, K.T.; Oo, W.; Su, T.T.; et al. Development and validation of a new short-form health literacy instrument (HLS-SF12) for the general public in six Asian countries. HLRP Health Lit. Res. Pr. 2019, 3, e91-e102. [CrossRef] [PubMed]

39. Duong, T.V.; Aringazina, A.; Baisunova, G.; Nurjanah; Pham, T.V.; Pham, K.M.; Truong, T.Q.; Nguyen, K.T.; Oo, W.; Mohamad, E.; et al. Measuring health literacy in Asia: Validation of the HLS-EU-Q47 survey tool in six Asian countries. J. Epidemiol. 2017, 27, 80-86. [CrossRef]

40. United Nations, Department of Economic and Social Affairs, Population Division. World Population Ageing 2015 (ST/ESA/SER.A/390); United Nations, Department of Economic and Social Affairs, Population Division: New York, NY, USA, 2015.

41. Department of Statistics Malaysia Official Website-Household Income and Basic Amenities Survey Report. 2019. Available online: https: / / www.dosm.gov.my (accessed on 14 July 2021).

42. IBM Corp. IBM SPSS Statistics for Windows, Version 27.0; IBM Corp: Armonk, NY, USA, 2020.

43. World Medical Association-Declaration of Helsinki: Ethical Principles for Medical Research Involving Human Subjects. Available online: https:/ / www.wma.net/policies-post/wma-declaration-ofhelsinki-ethical-principles-for-medical-researchinvolvinghuman-subjects / (accessed on 15 July 2021).

44. Storms, H.; Aertgeerts, B.; Vandenabeele, F.; Claes, N. General practitioners' predictions of their own patients' health literacy: A cross-sectional study in Belgium. BMJ Open 2019, 9, e029357. [CrossRef]

45. Abdullah, A.; Liew, S.M.; Ng, C.J.; Ambigapathy, S.; Paranthaman, P.V.V. Health literacy experiences of multi-ethnic patients and their health-care providers in the management of type 2 diabetes in Malaysia: A qualitative study. Health Expect. 2020, 23 , 1166-1176. [CrossRef]

46. Lausen, L.H.; Smith, S.K.; Cai, A.; Meiser, B.; Yanes, T.; Ahmad, R.; Rowlands, G. How is health literacy addressed in primary care? Strategies that general practitioners use to support patients. J. Commun. Health 2018, 11, 278-287. [CrossRef]

47. Speros, C.I. More than Words: Promoting health literacy in older adults. Am. Nurses Assoc. 2009, 14, 5.

48. McDougall, G.J.; Mackert, M.; Becker, H. Memory performance, health literacy, and instrumental activities of daily living of community residing older adults. Nurs. Res. 2012, 61, 70-75. [CrossRef]

49. Nakayama, K.; Osaka, W.; Togari, T.; Ishikawa, H.; Yonekura, Y.; Sekido, A.; Matsumoto, M. Comprehensive health literacy in Japan is lower than in Europe: A validated Japanese-language assessment of health literacy. BMC Public Health 2015, 15, 1-12. [CrossRef]

50. Esahak, A.I.; Juni, M.H.; Fuziah, P. Factors associated with health literacy among type 2 diabetes mellitus patients attending a government health clinic. Int. J. Public Health Clin. Sci. 2016, 3, 50-64.

51. Shahrir, S.N.; Shamsuddin, K.; Nor, N.S.M.; Man, C.S. Limited health literacy and its associated factors among overweight and obese housewives living in Klang valley low cost flats: Findings from the my body is fit and fabulous at home. Malays. J. Public Health Med. 2018, 18, 19-27.

52. Aydın, G.; Kaya, N.; Turan, N. The role of health literacy in access to online health information. Procedia Soc. Behav. Sci. 2015, 195, 1683-1687. [CrossRef]

53. Levin-Zamir, D.; Bertschi, I. Media health literacy, eHealth literacy, and the role of the social environment in Context. Int. J. Environ. Res. Public Health 2018, 15, 1643. [CrossRef] 
54. Manafo, E.; Wong, S. Health literacy programs for older adults: A systematic literature review. Health Educ. Res. 2012, 27, 947-960. [CrossRef] [PubMed]

55. Hersh, L.; Salzman, B.; Snyderman, D. Health literacy in primary care practice. Am. Fam. Physician 2015, 92, 118-124. [PubMed]

56. Hironaka, L.K.; Paasche-Orlow, M. The implications of health literacy on patient-provider communication. Arch. Dis. Child. 2008, 93, 428-432. [CrossRef] [PubMed]

57. Statistics and Data Intelligence Department-Internet Users Survey 2020: Malaysian Communications and Multimedia Commission. 2020. Available online: http:/ / www.mcmc.gov.my (accessed on 18 August 2021).

58. The Malaysian Administrative Modernisation and Management Planning Unit—High Speed Broadband: MyGOV Whole of Government. 2021. Available online: https://www.malaysia.gov.my/portal/content/30046 (accessed on 19 August 2021).

59. Statistics and Data Intelligence Department-Communications and Multimedia Pocket Book of Statistics Malaysia: Malaysian Communications and Multimedia Commission. 2020. Available online: http:/ / www.mcmc.gov.my (accessed on 18 August 2021).

60. Malaysian Communications and Multimedia Commission-Handphone User Survey 2018: Malaysian Communications and Multimedia Commission. 2018. Available online: http:/ / www.mcmc.gov.my (accessed on 18 August 2021).

61. Communications and Multimedia Consumer Forum of Malaysia. Complaints About Network and High-Speed Broadband (HSBB) Price Record an Increase in 2018. Available online: https:/ / consumerinfo.my/complaints-network-high-speed-broadbandincrease/ (accessed on 19 August 2021).

62. The Malaysian Administrative Modernisation and Management Planning Unit. The National Fiberisation and Connectivity Plan (NFCP). Available online: https:/ / www.malaysia.gov.my/portal/content/30736 (accessed on 19 August 2021).

63. World Health Organization Europe-Health Literacy the Solid Facts. The Regional Office for Europe of the World Health Organization. 2013. Available online: Htts:/ / www.euro.who.int/pubrequest (accessed on 19 August 2021). 